Christian Schaller

Nachhaltige Integration von Marketing und Innovieren 
GABLER EDITION WISSENSCHAFT 
Christian Schaller

\section{Nachhaltige Integration von Marketing und Innovieren}

Eine Theorie entwickelnde Longitudinalstudie zum Management des Wandels

Mit einem Geleitwort von PD Dr. Frank Piller

Deutscher Universitäts-Verlag 
Bibliografische Information Der Deutschen Nationalbibliothek

Die Deutsche Nationalbibliothek verzeichnet diese Publikation in der

Deutschen Nationalbibliografie; detaillierte bibliografische Daten sind im Internet über

<http://dnb.d-nb.de> abrufbar.

Dissertation Techn. Universität München, 2005

1. Auflage Januar 2007

Alle Rechte vorbehalten

(C) Deutscher Universitäts-Verlag I GWW Fachverlage GmbH, Wiesbaden 2007

Lektorat: Brigitte Siegel / Stefanie Loyal

Der Deutsche Universitäts-Verlag ist ein Unternehmen von Springer Science+Business Media. www.duv.de

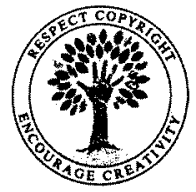

Das Werk einschließlich aller seiner Teile ist urheberrechtlich geschützt. Jede Verwertung außerhalb der engen Grenzen des Urheberrechtsgesetzes ist ohne Zustimmung des Verlags unzulässig und strafbar. Das gilt insbesondere für Vervielfältigungen, Übersetzungen, Mikroverfilmungen und die Einspeicherung und Verarbeitung in elektronischen Systemen.

Die Wiedergabe von Gebrauchsnamen, Handelsnamen, Warenbezeichnungen usw. in diesem Werk berechtigt auch ohne besondere Kennzeichnung nicht zu der Annahme, dass solche Namen im Sinne der Warenzeichen- und Markenschutz-Gesetzgebung als frei zu betrachten wären und daher von jedermann benutzt werden dürtten.

Umschlaggestaltung: Regine Zimmer, Dipl.-Designerin, Frankfurt/Main

Gedruckt auf säurefreiem und chlorfrei gebleichtem Papier

Printed in Germany

ISBN 978-3-8350-0518-1 


\section{Geleitwort}

Die Arbeit von Christian Schaller, die ich als Tutor am Lehrstuhl für Betriebswirtschaftslehre: Information, Organisation und Management an der Technischen Universität München betreuen durfte, diskutiert eine an sich klassische Thematik: Wie kann eine effektive Integration von Marketing und Innovationsmanagement erfolgen, eine Schnittstelle, die unisono als kritischer Erfolgsfaktor für den Unternehmenserfolg gilt. Gleichermaßen hat die bisherige Forschung oft ausgeprägte Disharmonien zwischen beiden Bereichen festgestellt. Mit zunehmend komplexer werdenden Märkten und Technologien wird die Spezialisierung dieser Funktionen weiter zunehmen - und damit die Bedeutung der Schnittstellenproblematik. Die Managementforschung beschäftigt sich seit den 1970er Jahren intensiv mit diesem Thema. Dabei hat sich das Forschungsinteresse von einem ursprünglich engen, funktional, instrumentell und innenorientierten Fokus heute zu einer eher ressourcen- und netzwerkorientierten Perspektive gewandelt.

An dieser Perspektive knüpft auch die Arbeit von Dr. Schaller an, die den Diskurs zur Schnittstellenthematik unter einer institutionellen, netzwerk- und beziehungsorientierten Perspektiv fortschreibt. These der Arbeit ist, dass sich dic Schwerpunkte im Marketing und Innovationsmanagement heute verlagert haben, so dass sich auch der Schwerpunkt der Integration beider Funktionen verlagert. Empirische Grundlage der Diskussion bildet eine longitudinale Einzelfallstudie auf Basis des "Grounded Theory"-Ansatzes. In einem Forschungsprozess von über vier Jahren hat Christian Schaller in ständiger Interaktion und Exploration mit dem Fallstudienunternehmen eine Theorie des Erwartungsmanagements zur nachhaltigen Integration von Marketing und Innovieren entwickelt.

Ergebnis dieses Forschungsprozesses sind wesentliche Beiträge zur Forschung über die Schnittstellenthematik:

- Die Arbeit entwickelt zur Untersuchung der Schnittstellenthematik ein neues, aktivitätsorientiertes, netzwerk- und beziehungsorientiertes Verständnis von Marketing und Innovieren, das in kohärenter Form auf ein institutionelles und prozessualdynamisches Verständnis von Organisation, Führung und Wandel übertragen wird.

- Die bestehende Diskussion im Bereich Beziehungsmarketing bezieht sich vor allem auf die effiziente und effektive Gestaltung operativer Austauschbeziehungen zwischen Anbieter und Abnehmer. Im Innovationsmanagement dagegen betrachten aktuelle Ansätze, die den Einbezug von Nutzern und Kunden in den Innovationsprozess fordern, diese Aktionen rein funktional, ohne dabei in der Regel auf die Art der Interaktion zwischen Kunde und Hersteller einzugehen. Christian Schaller integriert mit seiner Arbeit beide Perspektiven und leistet damit einen wesentlichen Beitrag zur Theorieentwicklung.

- Darüber hinaus leistet die Arbeit aber in der Betrachtung und Gestaltung des organisationalen Wandels in einem mittelständischen Unternehmen (vom Produkt- 
lieferanten zum integrierten Serviceanbieter) auch einen hoch praxisbezogenen Beitrag, der durch die innovative Forschungsmethodik unterstützt wird.

- Die Arbeit liefert aber auch aus methodischer Sicht ein willkommenes Beispiel methodischen Pluralismus. Der Autor verwendet "Grounded Theory Building" nicht nur als Deckmantel einer oberflächlichen explorativen Forschung, sondern demonstriert, dass auch qualitativ methodische Forschung hoch anspruchsvoll und methodisch fordernd sein kann. Anlage, Diskussion und Reflektion der empirischen Studie stellen eine eigenständige Leistung der Arbeit dar.

Insgesamt ist so die vorliegende Arbeit von Christian Schaller sowohl durch ein hohes theoretisches Niveau als auch durch eine hohe Relevanz für die Praxis gekennzeichnet, was ihre Lektüre gleichermaßen für Wissenschaftler wie Praktiker interessant und gewinnbringend macht. In diesem Sinne wünsche ich der Arbeit weite Verbreitung und Anerkennung in Wissenschaft und Praxis.

PD Dr. Frank Piller

MIT Sloan School of Management

Cambridge, MA (USA) 


\section{Vorwort}

Die vorliegende Dissertation entstand während meiner Tätigkeit als wissenschaftlicher Mitarbeiter am Lehrstuhl für Betriebswirtschaftslehre - Information, Organisation \& Management (IOM) an der Technischen Universität München (06/2000-01/2005). Beim Rückblick auf den mit dieser Forschungsarbeit verknüpften Lernprozess möchte ich zwei Aspekte besonders hervorheben. Der eine markiert den Anspruch, mit dem diese Forschungsreise gestartet war, dezidiert nach „rigor AND relevance“ streben zu wollen. Von Neugierde und Streben nach wissenschaftlich höchsten Standards geprägt, aber auch einer Verantwortung nach „managerial relevance“ und darüber hinaus gewahr. Der zweite Aspekt muss daher unweigerlich den partizipativ und pluralistischen Charakter dieses Forschungsprozesses betonen. Wie sollte obigem Anspruch besser begegnet werden können, als einerseits in ein Netzwerk an kompetenten WissenschaftlerInnen eingebunden und andererseits - auf Basis des gewählten Forschungsansatzes der ,in-depth research“ - mit einer von Vertrauen und Offenheit geprägten Beziehung zum gewählten Forschungspartner?

- Ersteres ist insbesondere geprägt durch den Doktorvater Prof. Dr. Dr. h.c. Ralf Reichwald, den Ko-Referenten Prof. Dr. Joachim Henkel, das Feedback von PD Dr. Frank Piller, die Methoden-Diskussionen mit Dipl.-Sozw. Oliver Brakel und Dipl.-Sozw. Andreas Tasch und ganz wesentlich durch die immer gesprächs- und hilfsbereiten Diskussionspartner in der Relationship Marketing-„,community“ wie Prof. Dr. Evert Gummesson, Prof. Dr. Richard Varey, Prof. Dr. Andreas Eggert, Prof. Dr. Florian Siems und Dr. Dominik Georgi. Jedoch auch für die Innovationsforschung Prof. Dr. Jürgen Hauschildt und für die Organisations- und Wandelforschung Prof. Dr. Georg Schreyögg und Dr. Judith Mühlbach gilt es hier zu erwähnen.

- Letzteres ist geprägt durch Michael Huber München (MHM) als Partnerorganisation für das Forschungsprojekt und den Geschäftsführer und Teil-Inhaber der Huber Gruppe Dipl.$\mathrm{Kfm}$. Heiner Ringer. Ohne ihn, sein Interesse an der Fragestellung, sein Vertrauen und seine vorbehaltlose Unterstützung, die einen uneingeschränkten Zutritt zu MHM und seinen Kunden und Partnern ermöglichte, hätte es dieses Projekt nicht gegeben. Frau Dipl.-Chem. Ursula Borgmann als ,gatekeeper“ für das Projekt und das gleichermaßen offene wie freundliche Entgegenkommen aller MitarbeiterInnen von MHM schufen dann die Basis, am Alltagsgeschehen von MHM in aller erforderlichen Nähe teilhaben zu können.

Ihnen allen wie auch den Personen aus dem Kreis von Familie und Freunden gilt mein Dank, mit größtmöglichem Nachdruck. 


\section{Inhaltsverzeichnis}

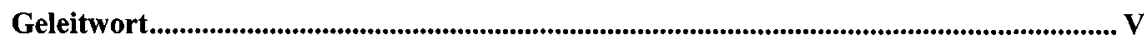

Vorwort .............................................................................................................................................. VII

Inhaltsverzeichnis.............................................................................................................IX

Abbildungsverzeichnis .............................................................................................................XIII

Tabellenverzeichnis ....................................................................................................... XVII

Abkürzungsverzeichnis...............................................................................................................XIX

1. Einleitung .................................................................................................................................. 1

1.1 Ausgangssituation, Problemstellung und Forschungslücken .................................... 2

1.2 Theoretische Fundierung, Forschungsansatz und Foschungsfrage des

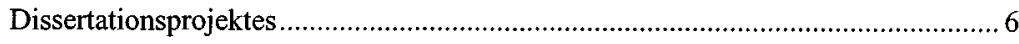

1.3 Vorgehensweise und Struktur der Arbeit ............................................................ 10

2. Dekonstruktion - ,root distinctions“ ${ }^{6 . . . . . . . . . . . . . . . . . . . . . . . . . . . . . . . . . . . . . . . . . . . . . . . . . . . . . . . . . . . . . . . . . . . . . . . . . . . . . . . . . . . . . . ~} 15$

2.1 Forschung im Kontext des Themas .............................................................. 15

2.1.1 Kritische Subjektivität und Empirische Pilotstudien ..................................... 16

2.1.2 Paradigmenwechsel im Marketing............................................................. 23

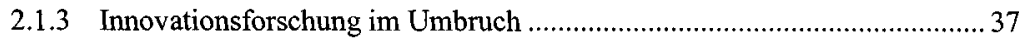

2.1.4 Forschungsintereresse und -antworten im Wandel .....................................50

2.1.4.1 Forschungsantworten der Marketingforschung.........................52

2.1.4.2 Forschungsantworten der Innovationsforschung..........................66

2.1.4.3 Stand der Forschung im Überblick............................................ 74

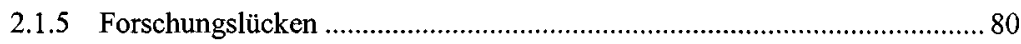

2.2 Wissenschaftstheoretische und methodologische Fundierung ............................. 85

2.2.1 Epistemologische Verankerung im interpretativen Paradigma..................... 85

2.2.2 Methodologische Einbettung in der qualitativen Sozialforschung ............... 90

2.2.3 Gütekriterien aus interpretativ-relationaler Perspektive ............................... 96

2.3 Theoretische Verortung und konzeptioneller Bezugsrahmen.............................. 102

2.3.1 „Relational network“-Theorie des Relationship Marketing ........................ 103

2.3.2 „Enactment theory“ des Organisierens ................................................... 109

2.3.3 „Open innovating"-Konzept des Innovationsmanagements ........................ 118

2.3.4 Theoretischer Bezugsrahmen................................................................ 123

2.4 Zusammenfassung der ,root distinctions“...................................................... 125 
3. Rekonstruktion - ,empirical journey“6 ................................................................... 127

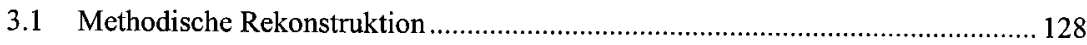

3.1.1 Theorieentwickelnde Forschungsstrategie der Empirie........................... 129

3.1.2 Forschungsansatz zwischen Prozess-, Aktions- und Fallstudien

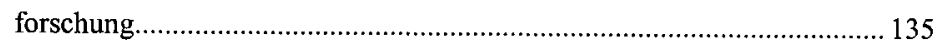

3.1.2.1 Longitudinale Prozessforschung als Forschungsansatz ........... 135

3.1.2.2 Abgrenzung und Anreicherung in Bezug zu Fallstudienund Aktionsforschung ...................................................... 140

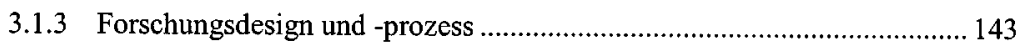

3.1.3.1 Wahl der Forschungspartner ................................................. 144

3.1.3.2 Methoden der Empirie ............................................................. 151

3.1.3.3 Dokumentation und Datenanalyse......................................... 158

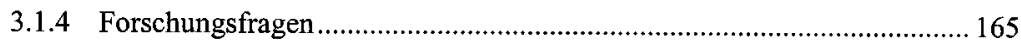

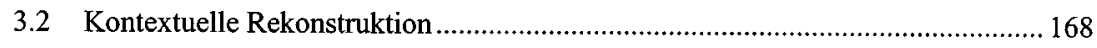

3.2.1 Mittelständische Industrie in Deutschland............................................. 168

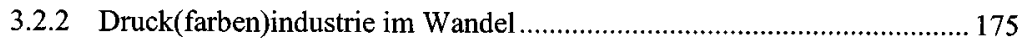

3.2.2.1 Druckindustrie im Wandel .................................................. 176

3.2.2.2 Druckfarbenindustrie im Wandel .......................................... 181

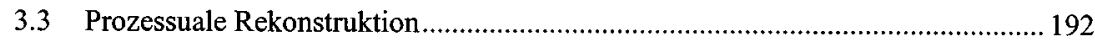

3.3.1 Huber Gruppe und Michael Huber München im Wandel......................... 194

3.3.1.1 Chronologie der Firmenentwicklung........................................ 194

3.3.1.2 Profil(e) und Strategie(n) in horizontaler Kontextualität .......... 197

3.3.2 Explorationsphase - ,breakpoint“"Vision 2000 als Wandelinitiative........ 202

3.3.2.1 Vision 2000 - Initiative zum nachhaltigen strategischen Wandel................................................................................ 204

3.3.2.2 Kultureller und struktureller Wandel mit der Vision 2000 ...... 209

3.3.2.3 Wandel zur Dienstleistungsführerschaft - (Teil-)Projekt „Service Excellence“.............................................................. 215

3.3.2.4 Relevante Themen im Fokus.................................................. 225

3.3.3 Vertiefungsphase - Integration von Marketing und Innovieren ................2230

3.3.3.1 Vertiefungsphase in ihrer Kontextualität ............................. 232

3.3.3.2 Marketingfunktion - Organisation und Beziehungen ............... 242

3.3.3.3 Innovierenfunktion - Organisation und Beziehungen.............. 257

3.3.3.4 Zentrale In-/Effektivitäten in Marketing- und Innovieren

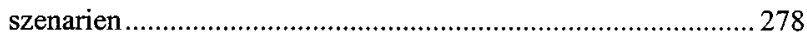

3.3.3.5 Resümee zur Vertiefungsphase ......................................... 303

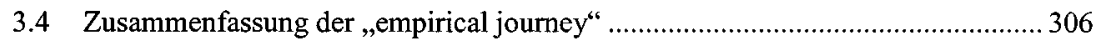


4. Konstruktion - ,disciplined integration"

4.1 In-/Effektivitäten in Marketing, Innovieren, deren Zusammenspiel und ihre ursächlichen Bedingungen

4.1.1 Interpretative Wende - In-/Effektivitäten in Marketing- und Innovierenszenarien ,revisited“.

4.1.2 „Interpretations of interpretations“ - ursächliche Bedingungen im Fokus.

4.1.3 Zusammenfassung des Zwischenstandes im Theorieentwicklungs prozess.

4.2 Wandel zur nachhaltigen Integration von Marketing und Innovieren

4.2.1 Gegenstandsbezogene Theorie im Kontext von Michael Huber München

4.2.1.1 In-/Effektivitäten der Integration von Marketing und Innovieren bei MHM

4.2.1.2 Kernkategorie „Erwartungsmanagement bei MHM im Beziehungsnetzwerk von Marketing und Innovieren“"

4.2.1.3 Gegenstandsbezogene Theorie des Erwartungsmanagements zur nachhaltigen Integration von Marketing und Innovieren bei MHM

4.2.2 Theorie des Erwartungsmanagements des Wandels zur nachhaltigen Integration von Marketing und Innovieren im industriellen Mittelstand... 386

4.3 Schlussfolgerungen für Theorie und Praxis 401

4.3.1 Theoretische und praxisbezogene Implikationen in Bezug zum Status quo der Diskurse

4.3.2 Beitrag zur Entwicklung einer (neuen) Marketingtheorie

4.4 Zusammenfassung der ,disciplined integration“.

5. Resümee und Ausblick

Anhang B: Empirie

B.1 Forschungsaktivitäten der Pilotstudien.

B.2 Forschungsaktivitäten der Empirie bei Michael Huber München

B.3 Verzeichnis der internen Dokumente aus der Sekundärdatenanalyse bei Michael Huber München

B.4 Leitfaden der ExpertInneninterviews in Marketing und Innovieren bei Michael Huber München 


\section{Abbildungsverzeichnis}

Abbildung 1: Vorgehensweise des Dissertationsprojekts und seiner empirischen Teilprojekte. 10

Abbildung 2: Modell zum Forschungsdesign ................................................................ 12

Abbildung 3: Struktur der Arbeit im Überblick ............................................................... 13

Abbildung 4: Zentrale Forschungsfelder und Diskurse im Fokus des Dissertationsprojekts.. 16

Abbildung 5: Ganzheitliches Modell zum Innovationsmanagement von Dienstleistungen.... 22

Abbildung 6: Paradigmenwandel im Marketing................................................................. 29

Abbildung 7: ,The market domain hierarchy“ ................................................................ 33

Abbildung 8: Ergebnisse einer Meta-Analyse von 19 empirischen Untersuchungen ............. 40

Abbildung 9: Ergebnisse einer Meta-Analyse von 44 empirischen Untersuchungen ............. 40

Abbildung 10: Ergebnisse einer Meta-Analyse von 60 empirischen Untersuchungen ...........41

Abbildung 11: Struktur der Darlegungen zum Stand der Forschung ...................................51

Abbildung 12: Problemdimensionen der Koordination zwischen Markt- und Betriebskurs eines Unternehmens....................................................................... 53

Abbildung 13: Forschungsinteresse im Wandel ............................................................. 82

Abbildung 14: Forschungslücken im Fokus ................................................................... 84

Abbildung 15: Gütekriterien zur Qualität der Forschung dieser Arbeit ............................... 97

Abbildung 16: ,Synthesis of specific theories and experiences into a more general marketing theory"

Abbildung 17: Marketingtheoretisches Fundament im Kontext der theorieentwickelnden

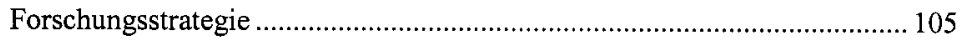

Abbildung 18: ,Relational network"-Theorie - marketingtheoretisches Verständnis dieser Arbeit ..................................................................................... 106

Abbildung 19: „Complete marketing equilibrium“ “.................................................... 108

Abbildung 20: Das Dualproblem aus Differenzierung und Integration .............................. 110

Abbildung 21: „Enactment theory“ - organisationstheoretisches Verständnis dieser

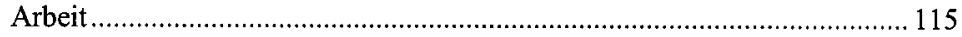

Abbildung 22: Quellen des innovationstheoretischen Verständnisses ............................ 119

Abbildung 23: „Open innovating"-Konzept - innovationstheoretisches Verständnis dieser Arbeit .................................................................................. 120

Abbildung 24: Theoretischer Bezugsrahmen im Überblick .......................................... 123

Abbildung 25: Ansatz der theorieentwickelnden Konstruktionsstrategie für diese Arbeit ... 134

Abbildung 26: Kernelemente der longitudinalen Prozessforschung ................................. 136

Abbildung 27: Methoden und Validität des Forschungsdesigns im Fokus ......................... 144

Abbildung 28: ,Sampling“ der Explorationsphase bei MHM............................................. 146

Abbildung 29: Initialer ,sampling“-Rahmen der Vertiefungsphase bei MHM.................... 147

Abbildung 30: Zeit-,,sampling“ der longitudinalen Prozessforschung bei MHM ................ 149 
Abbildung 31: Methoden der Empirie in Explorations-, Vertiefungs- und

Abschlussphase.

Abbildung 32: Struktur des Leitfadens für die ExpertInneninterviews der

Vertiefungsphase

Abbildung 33: Prozessschritte und Methoden bei Datenanalyse und Theorieentwicklung .. 161

Abbildung 34: Forschungsfragen im Detail ................................................................... 167

Abbildung 35: Wirtschaftliche Entwicklung der Druckindustrie in Deutschland ................ 177

Abbildung 36: Entwicklung der Absatzmengen und -werte von Druckfarben in

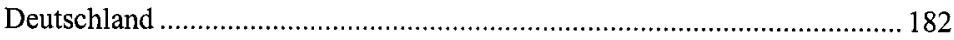

Abbildung 37: Absatzvolumina von Produkten der Druckfarbenindustrie in 2002 _............ 183

Abbildung 38: Bestandteile der Gesamtkosten eines Druckproduktes............................... 184

Abbildung 39: Die führenden Druckfarbenhersteller in Europa ......................................... 186

Abbildung 40: Prozessschritte der Datenanalyse und Theorieentwicklung in

Abschnitt 3.3.

Abbildung 41: In Abschnitt 3.3 adressierte Forschungsfragen........................................ 193

Abbildung 42: Zentrale Wandelphasen in der Entwicklung der Huber Gruppe ................. 198

Abbildung 43: Forschungsstrategie der Explorationsphase .............................................. 202

Abbildung 44: Aktionsstränge der Empirie der Explorationsphase ...................................... 203

Abbildung 45: Führungsgrundsätze in der Diskussion bei MHM ...................................... 210

Abbildung 46: Angestrebter kultureller Wandel im Zuge der Vision 2000 ......................... 211

Abbildung 47: „Organisation 2001“ als initiale strukturelle Umsetzung der Vision 2000 ... 212

Abbildung 48: Phasen eines vom LAT gesteuerten E-Projektes ....................................... 214

Abbildung 49: Phasen eines vom LAM gesteuerten (M-)Projektes .................................. 215

Abbildung 50: Ansatz des Teilprojektes ,Service Excellence“.......................................... 217

Abbildung 51: Forschungsinteresses als relevantes Thema aus Sicht der Organisation ....... 228

Abbildung 52: Forschungsstrategie der Vertiefungsphase ............................................... 231

Abbildung 53: Design des deskriptiven Teils der Vertiefungsphase................................... 232

Abbildung 54: Zusammenspiel zentraler Marketing-Teilfunktionsbereiche (I/II) .............. 250

Abbildung 55: Zusammenspiel zentraler Marketing-Teilfunktionsbereiche (II/II) .............. 251

Abbildung 56: Status quo der Organisation des Funktionsbereichs Innovieren auf

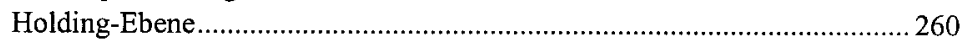

Abbildung 57: Zusammenspiel zentraler Innovieren-Parteien (I/II) ................................ 265

Abbildung 58: Zusammenspiel zentraler Innovieren-Parteien (II/II) ................................ 269

Abbildung 59: Zusammenspiel zentraler Parteien im Marketing- und Innovieren-

Szenario

Abbildung 60: In-/Effektivitäten im Marketing- und Innovieren-Szenario und deren

Zusammenspiel .................................................................................... 305

Abbildung 61: Prozessschritte der Datenanalyse und Theorieentwicklung in Kapitel 4 ...... 309

Abbildung 62: In Kapitel 4 adressierte Forschungsfragen 
Abbildung 63: Recodierte und konzeptualisierte zentrale In-/Effektivitäten in Marketing und Innovieren und deren Zusammenspiel aus Sicht des Forschers

Abbildung 64: Codierparadigma zum axialen Codieren

Abbildung 65: Kategorie „Kein funktionerendes Marketing“ im Beziehungsnetzwerk ....... 322

Abbildung 66: Kategorie „Zu viel Kundenparadies“ im Beziehungsnetzwerk

Abbildung 67: Kategorie „Reaktives Problemlösen als Innovieren“ im Beziehungsnetzwerk.

Abbildung 68: Kategorie „Projektmatrix ohne Führung und Dualorientierung“ im

Beziehungsnetzwerk.

Abbildung 69: Kategorie „Halblebige Umsetzung technischer Realisierungen“ im

Beziehungsnetzwerk.

Abbildung 70: Kategorie „Widerstände gegen das Führen von Wandel“ im

Beziehungsnetzwerk.

Abbildung 71: Zwischenstand im Theorieentwicklungsprozess auf Basis des eingesetzten Codierparadigmas

Abbildung 72: Das erweiterte Codierparadigma mit Fokus auf zentrale In-/Effektivitäten der Integration von Marketing und Innovieren sowie die Kernkategorie der ursächlichen Bedingungen und ihre Kernprozesse

Abbildung 73: Codierparadigma der In-/Effektivitäten im Prozess der zunehmenden Fokussierung.

Abbildung 74: Marketing und Innovieren bei MHM in Bezug zur Entwicklung der Disziplinen.

Abbildung 75: Status quo von Marketing und Innovieren bei MHM 336

Abbildung 76: Ebene der funktionalen Integration bei MHM

Abbildung 77: Ebene der prozessualen Integration bei MHM 338

Abbildung 78: Ebene der aktivitätsbasierten Integration bei MHM

Abbildung 79: Ebene der Integration aus einer netzwerkartigen Beziehungssicht von Erwartungskontexten bei MHM

Abbildung 80: Kern- und Kontext-In-/Effektivitäten der Integration von Marketing und Innovieren bei MHM

Abbildung 81: Kern-In-/Effektivitäten der Integration von Marketing und Innovieren bei MHM im Beziehungsnetz des Codierparadigmas

Abbildung 82: Selektives Codieren zur Entwicklung von Kernkategorie und konstituierender Prozesse

Abbildung 83: Einbettung der Kernkategorie in das Beziehungsnetz des

Codierparadigmas

Abbildung 84: Sinngebungs- und Institutionalisierungsprozesse der Kernkategorie des Erwartungsmanagements bei MHM

Abbildung 85: Ansatz zum Wandelprozess des Integrierens von Marketing und Innovieren bei MHM 
Abbildung 86: Zielparteien und Wandelorte des strategischen Wandels zum Integrieren von Marketing und Innovieren bei MHM

Abbildung 87: Status quo der Integration von Marketing und Innovieren bei MHM. 360

Abbildung 88: Zentrale Hemmnisse zum Wandel zur nachhaltigen Integration von Marketing und Innovieren bei MHM

Abbildung 89: Wandelkonzept zum Management der nachhaltigen Integration von Marketing und Innovieren bei MHM

Abbildung 90: Prozessuale Stablisierung über Rekalibrierung und ,sensemaking“ beim Wandel zur nachhaltigen Integration von Marketing und Innovieren bei MHM.

Abbildung 91: Gegenstandsbezogene Theorie des Erwartungsmanagements zur nachhaltigen Integration von Marketing und Innovieren bei MHM

Abbildung 92: Ebenen und Dimensionen der gegenstandsbezogenen Theorie des Erwartungsmanagements

Abbildung 93: Zentrale Wandelorte der gegenstandsbezogenen Theorie des Erwartungsmanagements

Abbildung 94: Mehrdeutigskeitsreduktion im Zentrum der Theorie zum Erwartungsmanagement

Abbildung 95: „Middle-range substantive theory“ des Erwartungsmanagements zur nachhaltigen Integration von Marketing und Innovieren im industriellen Mittelstand

Abbildung 96: Theoretischer und praxisbezogener Erkenntnisbeitrag in Bezug zum Status quo in Forschung und Praxis

Abbildung 97: Forschungsbedarfe im hermeneutischen Wandelprozess der Diskurse zum Forschungsinteresse

Abbildung 98: Beitrag der Erkenntnisse dieser Arbeit zum Prozess der Entwicklung der ,general theory“ des Relationship Marketing 


\section{Tabellenverzeichnis}

Tabelle 1: Beispielhafte Kommentare der Marketing- und Innovationsforschung zu Relevanz und Status quo der Schnittstelle zwischen Marketing und Innovieren

Tabelle 2: Entwicklung der Forschungsaktivitäten im Bereich NSD .................................... 42

Tabelle 3: Wandel im Innovationsmanagement ............................................................... 49

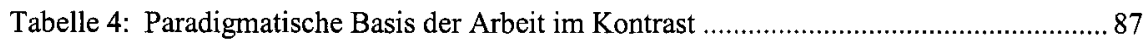

Tabelle 5: Methodologische Einbettung in der qualitativen Sozialforschung im Kontrast ....91

Tabelle 6: Charakterisierung der Unternehmensführung von Klein- und Mittelbetrieben ... 172

Tabelle 7: Charakterisierung der Forschung und Entwicklung in Klein- und

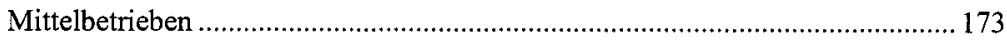

Tabelle 8: Umsätze der deutschen Druckindustrie nach Erzeugnissen/Leistungen .............. 179

Tabelle 9: Die führenden Druckfarbenhersteller weltweit 2003 .......................................... 187

Tabelle 10: Firmenchronologie von MHM und der Huber Gruppe ...................................... 196

Tabelle 11: Bisherige Untersuchungen der Marketingforschung zum Forschungsinteresse im Überblick.

Tabelle 12: Bisherige Untersuchungen der Innovationsforschung zum Forschungsinteresse

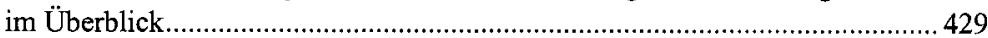

Tabelle 13: Forschungsaktivitäten der Explorationsphase ................................................. 431

Tabelle 14: Forschungsaktivitäten der Vertiefungsphase ....................................................433

Tabelle 15: Forschungsaktivitäten der Abschlussphase ....................................................... 433 


\section{Abkürzungsverzeichnis}

$\begin{array}{ll}\text { AMA } & \text { American Marketing Association } \\ \text { AWT } & \text { Anwendungstechnik } \\ \text { Anm. d. Verf. } & \text { Anmerkung des Verfassers } \\ \text { B2B } & \text { Business to business } \\ \text { B2C } & \text { Business to customer } \\ \text { BIP } & \text { Bruttoinlandsprodukt } \\ \text { BVDM } & \text { Bundesverband Druck und Medien } \\ \text { CEPE } & \text { European Council of the Paint, Printing Ink and Artists' Colours Industry } \\ \text { DFG } & \text { Deutsche Forschungsgemeinschaft } \\ \text { Drupa } & \text { Weltgrößte Messe im Bereich ,print media, publishing \& converting“ } \\ \text { EU } & \text { Europäische Union } \\ \text { EuPIA } & \text { European Printing Ink Association } \\ \text { FN } & \text { Fußnote } \\ \text { F\&E } & \text { Forschung \& Entwicklung } \\ \text { GF } & \text { Geschäftsführung } \\ \text { Herv. i. Orig. } & \text { Hervorhebung im Original } \\ \text { Hrsg. } & \text { HerausgeberIn } \\ \text { Huber } & \text { Michael Huber München GmbH / huber group } \\ \text { IA } & \text { Ink Academy } \\ \text { IfM } & \text { Institut für Mittelstandsforschung, Bonn } \\ \text { IMP } & \text { Industrial Marketing and Purchasing (Group) } \\ \text { IOM } & \text { Institut für Information, Organisation \& Management } \\ \text { KA } & \text { Key Account } \\ \text { KAD } & \text { Kaufmännischer Außendienst } \\ \text { KAM } & \text { Key Account Management } \\ \text { KfW } & \text { Kreditanstalt für Wiederaufbau } \\ \text { KMU } & \text { kleine und mittlere Unternehmen } \\ \text { LAM } & \text { Lenkungsausschuss Marketing } \\ \text { LAT } & \text { Lenkungsausschuss Technik } \\ \text { MHM } & \text { Michael Huber München GmbH } \\ \text { n. a. } & \text { not assigned } \\ \text { NPD } & \text { New Product Development } \\ \text { NSD } & \text { New Service Development } \\ \text { QFD } & \text { Quality Function Deployment } \\ \text { R\&D } & \text { Research \& Development } \\ \text { RM } & \text { Relationship Marketing } \\ \text { SME } & \text { Small and medium enterprises } \\ & \end{array}$


TAD Technischer Außendienst

TIM Technologie- und Innovationsmanagement

USD US-Dollar

VdMi Verband der Druckfarbenindustrie 